\title{
Understanding galaxy formation in the reionization era using the FIRE simulations
}

\author{
Xiangcheng $\mathrm{Ma}$ (1) \\ Department of Astronomy and Theoretical Astrophysics Center, \\ University of California Berkeley, Berkeley, CA 94720, USA \\ email: xchma@berkeley.edu
}

\begin{abstract}
We present a suite of high-resolution cosmological zoom-in simulations of galaxies at $z \geqslant 5$ using the state-of-the-art models for the multi-phase ISM, star formation, and stellar feedback from the FIRE project. We present a series of key results from these simulations, including the stellar mass-halo mass relation, the ultraviolet luminosity functions, dust attenuation and dust temperatures, the ubiquitous formation of bound star clusters, morphology and clumpiness, and the escape fractions of ionizing photons from high-redshift galaxies. We discuss how different simulations in the literature agree and disagree and what observations are most useful for testing the models in the era of ALMA and JWST.
\end{abstract}

Keywords. binaries: general, stars: formation, dust, extinction, ISM: structure, galaxies: evolution, galaxies: formation, galaxies: high-redshift, galaxies: star clusters, cosmology: theory

\section{Introduction}

Understanding the formation of galaxies in the first billion years of the Universe is one of the most important questions in extragalactic astronomy and cosmology. It is believed that these high-redshift galaxies are the dominant sources for cosmic reionization (e.g., Robertson et al. 2013). Moreover, they open a new window for testing our understanding of galaxy formation physics. Limited by the capability of current observational facilities, our knowledge of high-redshift galaxies to date is far less extensive than those at low and intermediate redshifts. Thanks to the Atacama Large Millimeter Array (ALMA) and the upcoming James Webb Space Telescope (JWST), we expect to collect enormous data of galaxies at $z \gtrsim 5$ with unprecedented quality over the next few years. It is thus critical and timely, from a theoretical point of view, to make more detailed, realistic predictions for high-redshift galaxies that can be confronted with future observations.

We will present a sample of high-resolution, cosmological hydrodynamic zoom-in simulations of high-redshift galaxies we have been building for the past $\sim 2-3$ years. These simulations adopt the Feedback in Realistic Environments (FIRE) models for the multi-phase interstellar medium (ISM), star formation, and stellar feedback (Hopkins et al. 2018). Our simulations complement the semi-analytic models (e.g., Yung et al. 2019) and large-volume cosmological simulations of high-redshift galaxies (e.g., Feng et al. 2016) by resolving small-scale structures and physical processes inside the galaxies. We note that the FIRE project also has a large simulation library run to $z=0$ and $z=2$, which other zoom-in simulations of high-redshift galaxies may not have (e.g., Pallottini et al. 2017). We will briefly describe the simulation sample and present the key results we have published. We will also discuss in what aspects simulations and models from different groups agree and disagree and what future observations can be used to test these predictions. 


\section{The simulations}

These simulations use the well-established multi-scale cosmological zoom-in technique. In short, a dark-matter-only cosmological volume is simulated first. Halos of desired mass at desired redshift are selected. The region around the target halos is then re-simulated with baryons at much higher resolution, while the rest of the box is simulated at lower resolution to keep the large-scale tidal force. Our high-redshift simulation sample consists of 35 zoom-in regions run to $z=5-9$ selected from a $(120 \mathrm{Mpc} / h)^{3}$ box. The baryonic mass and spatial resolution adopted in these simulations are $\sim 100-7000 M_{\odot}$ and $\sim 0.1-1 \mathrm{pc}$. The entire sample contains over $\sim 2000$ halos from $M_{\text {halo }} \sim 10^{8}-10^{12} M_{\odot}$ in all the zoomin regions. We refer to Ma et al. (2019a) for details of the initial conditions.

These simulations use the FIRE-2 model for the multi-phase ISM, star formation, and feedback, which we briefly summarize here. Gas cooling is followed from $10-10^{10} \mathrm{~K}$, including free-free, bound-free, atomic, and metallicity-dependent metal-line, finestructure, and molecular cooling. Star formation is restricted to molecular, selfgravitating gas with density above $10^{3} \mathrm{~cm}^{-3}$. The model also includes explicit treatments for photoionization, photo-heating, radiation pressure, stellar winds, and supernovae feedback. We consider metal enrichment from Type-II, Type-Ia SNe, and AGB winds and subresolution metal mixing. We refer to Hopkins et al. (2018) for details of the FIRE-2 model.

\section{Results}

We highlight some of the key results based on the high-redshift galaxy simulation suite from the FIRE project, but refer to the papers we have published in recent years for more details (Ma et al. 2015, 2016, 2018a,b, 2019a,b).

The stellar mass-halo mass (SMHM) relation at $z \gtrsim 5$. We find the SMHM relation for our simulated galaxies can be best described by $\log M_{*}=1.58\left(\log M_{\text {halo }}-10\right)+7.1$ for halo mass $M_{\text {halo }} \sim 10^{8}-10^{12} M_{\odot}$ with little evolution in $z \sim 5-12$. We stress that the redshift evolution of the SMHM relation is still an open question. Very different trends have been reported among cosmological simulations, semi-analytic models, and empirical models (e.g., Behroozi \& Silk 2015; Ceverino et al. 2017; Wilkins et al. 2017; Tacchella et al. 2018). The discrepancy can be resolved by better stellar mass measurements with improved data in the rest-frame optical bands from JWST.

Stellar mass functions (SMFs) and luminosity functions (LFs). In Ma et al. (2018a), we derive the SMFs and multi-band LFs from our simulations. The left panel of Figure 1 shows the rest-frame UV (1500 $)$ LF at $z=6$, where we compare our results (dashed lines) with most up-to-date observational constraints (color points). The bright-end UV LFs $\left(\mathrm{M}_{\mathrm{UV}}<-17\right)$ up to $z \sim 8$ are the most well constrained properties of highredshift galaxies. Our simulations agree with observations very well, especially when dust attenuation is taken into account. We refer to Ma et al. (2018a) for more results.

Dust attenuation, dust emission, and dust temperature. We have conducted full threedimensional Monte Carlo dust radiative transfer calculations on our simulations in Ma et al. (2019a), using the public SKIRT code (Baes et al. 2011). We reiterate dust attenuation is required for shaping the bright-end UVLFs even at $z \sim 8$. The right panel of Figure 1 shows the relation between peak wavelength of dust emission, $\lambda_{\text {peak }}$, and the total dust emission, $L_{\mathrm{IR}}$. The color points represent individual galaxies at $z \geqslant 5$ from our simulated sample, while the red triangles show the $z=0$ FIRE simulations for reference. The grey points, orange lines, and blue squares show the observed data at lower redshifts compiled by Casey et al. (2018). We find $\lambda_{\text {peak }}$ decreases with redshift at fixed $L_{\mathrm{IR}}$, which implies higher dust temperatures at higher redshifts. This can be understood by higher specific star formation rates and higher star formation surface densities at higher 

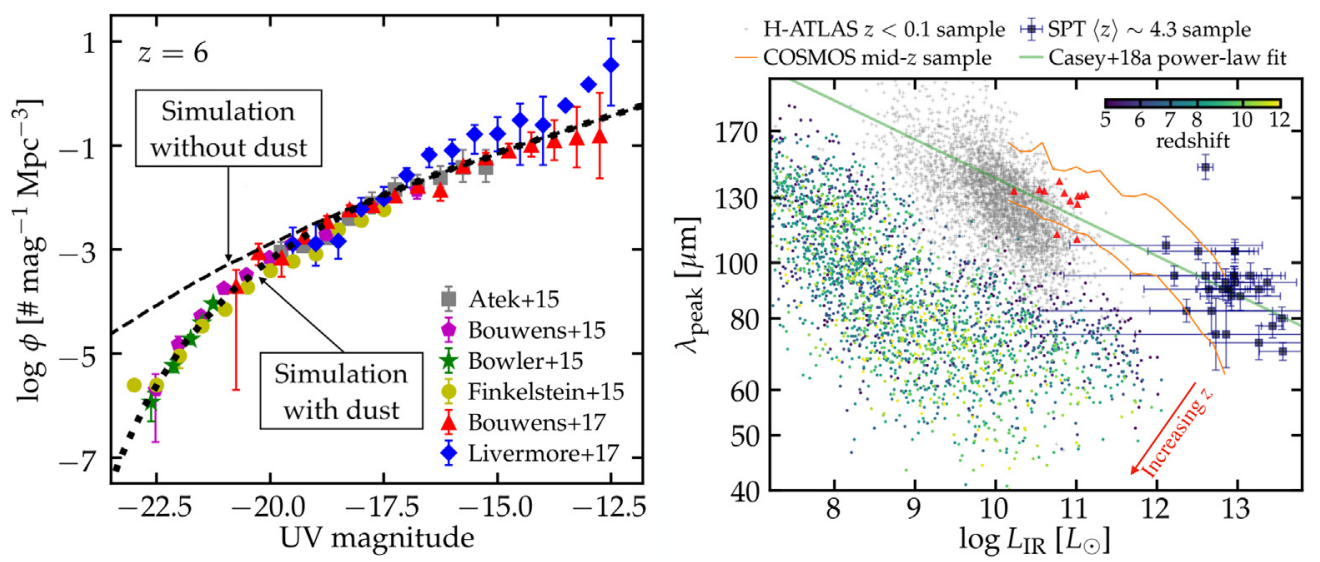

Figure 1. Left: The UVLF at $z=6$. The dashed lines show predictions from our simulations, with (thick) and without (thin) dust attenuation. The color points show observational constraints from the literature. Right: The relation between peak wavelength of dust emission, $\lambda_{\text {peak }}$, and the total dust emission, $L_{\mathrm{IR}}$.

redshifts. We refer to Ma et al. (2019a) for more results (e.g., the IRX- $\beta$ relation), but emphasize the importance for distinguishing different definitions of dust temperature.

Ubiquitous star cluster formation. We identify a large number of bound star clusters in almost all of our simulations of high-redshift galaxies. In Ma et al. (2019b), we investigate how these star clusters form in these simulations by re-simulating a starburst in three galaxies spanning over three orders of magnitude in stellar mass with much finer output frequency. Figure 2 shows the formation sites of two clusters in a galaxy of $M_{*} \sim 10^{9} M_{\odot}$ (halo mass $M_{\text {halo }} \sim 10^{11} M_{\odot}$ ) at $z \sim 5$. In general, high-redshift galaxies are highly gasrich and turbulent. High-pressure clouds form efficiently in such environments via cloudcloud collision and compression by feedback-driven outflows. Stars form in these clouds at near unity efficiency within a cloud free-fall time, so they tend to stay gravitationally bound at formation. We find that newly formed clusters broadly follow a power-law mass function of $\mathrm{d} N / \mathrm{d} M \propto M^{-2}$ (see Ma et al. 2019b for more details).

Clumpy morphologies. As high-redshift galaxies tend to form a large number of star clusters, one implication is that their rest-frame UV morphologies are very clumpy. The left panel of Figure 3 shows one example galaxy from our simulated sample, which has stellar mass $M_{*} \sim 2 \times 10^{8} M_{\odot}$ and $\mathrm{UV}$ magnitude $\mathrm{M}_{\mathrm{UV}} \sim-16.7$. The rest-frame $\mathrm{UV}$ image is dominated by three star-forming clumps that do not contribute a large stellar mass. For observations at finite surface brightness depth, one may miss most of the UV light but only see the brightest clump. The right panel of Figure 3 illustrates this effect. We caution that the faint galaxies detected in the Hubble Frontier Fields are likely biased by individual star-forming clumps. This makes it more difficult to constrain the faint-end UVLFs robustly at high redshifts.

Escape fractions of ionizing photons from high-redshift galaxies. In Ma et al. (2015, 2016), we have conducted Monte Carlo radiative transfer calculations of Lymancontinuum photons on a small sample of high-redshift galaxy simulations run with the previous version of the FIRE model to study the escape fraction of hydrogen ionizing photons, $f_{\text {esc }}$, from $z \geqslant 5$ galaxies. We find one key physics that determines $f_{\text {esc }}$ is the competition of time-scales between birth cloud disruption and stellar evolution: most ionizing photons cannot escape before feedback from young stars disrupt and clear their birth clouds, but if this happens longer than a few Myrs, there will be fewer ionizing photons available as massive stars have left the main sequence. We find if binary stars 

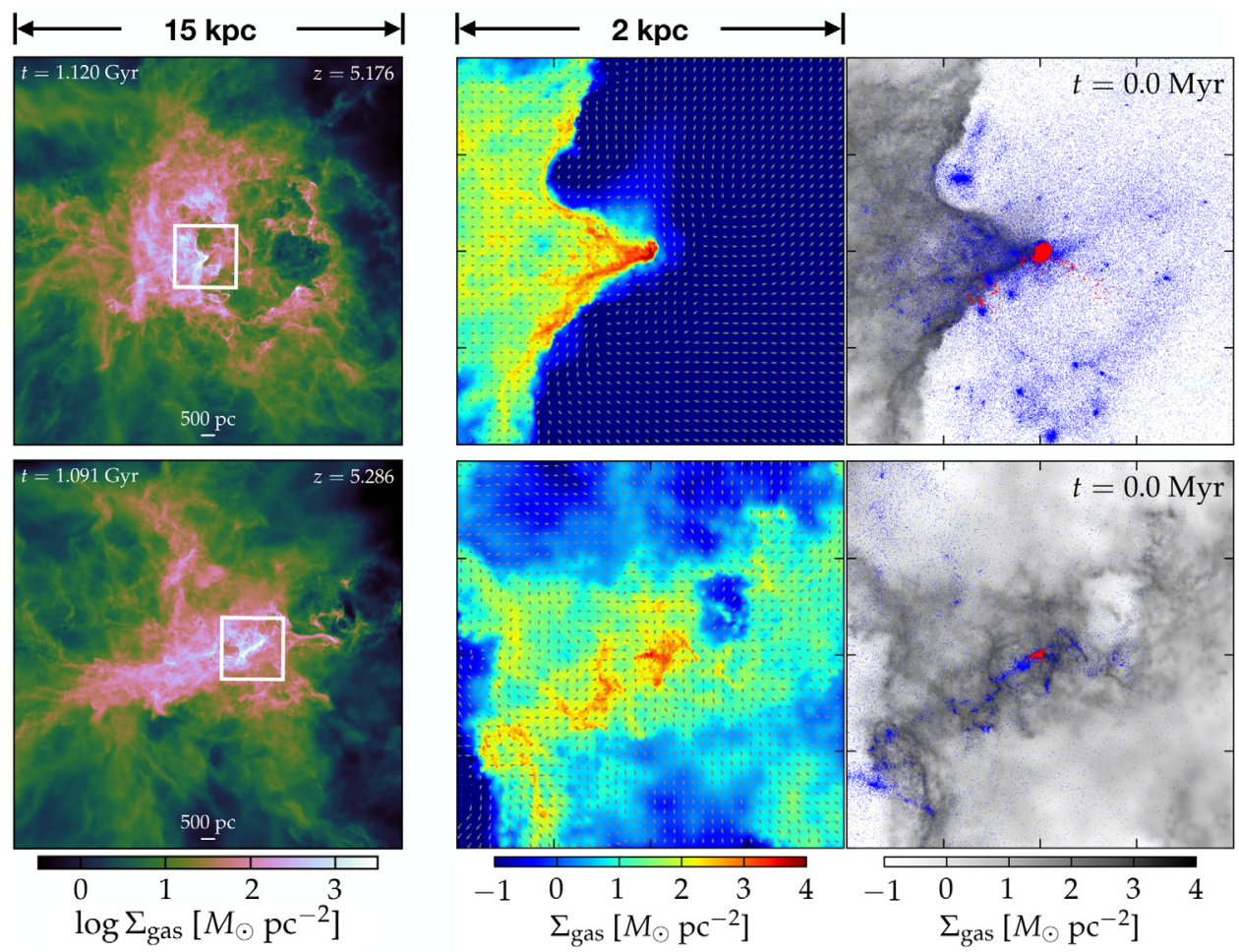

Figure 2. Bound star clusters formed in a simulated galaxy with stellar mass $M_{*} \sim 10^{9} M_{\odot}$ (halo mass $M_{\text {halo }} \sim 10^{11} M_{\odot}$ ) at $z \sim 5$. These clusters form in high-pressure clouds with gas surface density $\Sigma_{\text {gas }} \gg 10^{3} M_{\odot} \mathrm{pc}^{-2}$, which are formed in highly gas-rich, turbulent ISM via cloud-cloud collision and compression by feedback-driven winds.
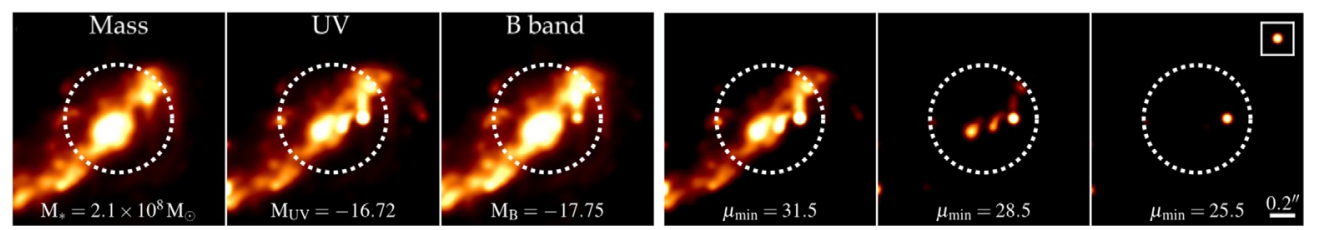

Figure 3. Left: Stellar mass, rest-frame UV, and optical maps of a simulated galaxy in our sample at $z \sim 6$. The UV image is dominated by three compact star-forming clumps that do not contribute much stellar mass. Right: Rest-frame UV images when the galaxy is observed at finite surface brightness depths.

are taken into account, $f_{\text {esc }}$ will increase by a large factor, as binaries produce more ionizing photons on $10 \mathrm{Myr}$ time-scales, which can escape from the galaxy much more easily.

\section{Discussion}

In the era of ALMA and JWST, there will be tremendous amount of new data coming in the next few years probing various properties of high-redshift galaxies that we cannot do in the past. We argue that such data make it possible to use high-redshift galaxies to test our understanding of galaxy formation physics.

First of all, we iterate the discrepancy on the SMHM relation and its redshift evolution between different theoretical predictions in the literature. The SMFs at $z \geqslant 5$ have not 
been well constrained so far (the discrepancy can be as large as an order of magnitude; e.g., Stefanon et al. 2017), partly due to the lack of high-quality data in the rest-frame optical bands. Better measurements on the stellar mass of high-redshift galaxies can be useful for testing the underlying assumptions (e.g., star formation efficiencies, feedback, the scatter of the SMHM relation, etc.) adopted in these models.

We find in our simulations, the galaxies are highly gas-rich and turbulence supported, even in halos as massive as $M_{\text {halo }} \sim 10^{12} M_{\odot}$ ( stellar mass up to $\left.M_{*} \sim 2 \times 10^{10} M_{\odot}\right)$. In such systems, star formation is bursty, star clusters form efficiently, and feedback is sufficiently strong to blow out nearly all the gas from the galaxy. There is no rotationally supported gas disk formed in these galaxies. Although such features are broadly in line with some work in the literature, we notice other simulations may produce very different galaxy properties (e.g., rotating disks form at very high redshift even in relatively lowermass halos; see Pallottini et al. 2017; Katz et al. 2019). The discrepancy is largely due to the star formation and feedback models adopted in these simulations. We propose the following observational diagnostics that can be used to test the simulations in the era of ALMA and JWST: H $\alpha$ - and UV-derived SFRs, Balmer break (as indicators of bursty star formation), morphology and clumpiness of high-redshift galaxies (to test star cluster formation), and gas kinematics measurements (cold gas from [C II] and ionized gas from Balmer lines, as diagnostics of rotational support). It would be extremely interesting to quantify burstiness and disk fraction as a function of stellar mass and redshift to form a coherent picture of galaxy formation across cosmic time. JWST and ALMA can definitely fill in the relatively massive at $z \gtrsim 6$.

\section{References}

Baes, M., Verstappen, J., De Looze, I., et al. 2011, ApJS, 196, 22

Behroozi, P. S. \& Silk, J., 2015, ApJ, 799, 32

Casey, C. M., Zavala, J. A., Spilker, J., et al. 2018, ApJ, 862, 77

Ceverino, D., Glover, S. C. O., \& Klessen, R. S., 2017, MNRAS, 470, 2791

Feng, Y., Di-Matteo, T., Croft, R. A., et al. 2016, MNRAS, 455, 2778

Hopkins, P. F., Wetzel, A., Kereš, D., et al. 2018, MNRAS, 480, 800

Katz, H., Kimm, T., Haehnelt, M. G., et al. 2019, MNRAS, 483, 2019

Ma, X., Kasen, D., Hopkins, P. F., et al. 2015, MNRAS, 453, 960

Ma, X., Hopkins, P. F., Kasen, D., et al. 2016, MNRAS, 459, 3614

Ma, X., Hopkins, P. F., Garrison-Kimmel, S., et al. 2018a, MNRAS, 478, 1694

Ma, X., Hopkins, P. F., Boylan-Kolchin, M., et al. 2018b, MNRAS, 477, 219

Ma, X., Hayward, C. C., Casey, C. M., et al. 2019a, MNRAS, 487, 1844

Ma, X., Grudić, M. Y., Quataert, E., et al. 2019b, arXiv e-print: 1906.11261

Pallottini, A., Ferrara, A., Gallerani, S., et al. 2017, MNRAS, 465, 2540

Robertson, B. E., Furlanetto, S. R., Schneider, E., et al. 2013, ApJ, 768, 71

Stefanon, M., Bouwens, R. J., Labbé, I., et al. 2017, ApJ, 843, 36

Tacchella, S., Bose, S., Conroy, C., et al. 2018, ApJ, 868, 92

Wilkins, S. M., Feng, Y., Di Matteo, T., et al. 2017, MNRAS, 469, 2517

Yung, L. Y. A., Somerville, R. S., Finkelstein, S. L., et al. 2019, MNRAS, 483, 2983 